\title{
EL AUGE Y DECLIVE DEL INSTITUTO \\ NACIONAL DE NUTRIOLOGÍA DE MÉXICO \\ Y SU PROYECTO DE NUTRICIÓN SOCIAL \\ DE 1943 A 1956
}

\author{
Joel Vargas Domínguez \\ Universidad Nacional Autónoma de México
}

INTRODUCCIÓN

G 1 doctor Salvador Zubirán Anchondo (1898-1998) es reconocido por haber dirigido uno de los más grandes centros de investigación en México, el Instituto Nacional de Ciencias Médicas y Nutrición, que a la postre llevaría su nombre. En sus memorias, el doctor Zubirán recordaba con especial agradecimiento a tres médicos que lo habían marcado en su formación: Gastón Melo, Manuel Gea González y Francisco de Paula Miranda (1890-1950). ${ }^{1}$ Los dos primeros han sido reconocidos por la historia de la medicina y la salud pública como impulsores de la modernización de la medicina en México en las décadas que siguieron a la revolución mexicana, pero Miranda ha sido poco abordado por la historiografía. Esta omisión quizá tiene que ver con una confusión que ha permeado la historiografía.

Fecha de recepción: 26 de julio de 2018

Fecha de aceptación: 8 de febrero de 2019

${ }_{1}$ Zubirán, Mi vida y mi lucha, p. 31. 
El Instituto Nacional de Nutrición a cargo de Zubirán, adquirió ese nombre hacia finales de la década de 1950. Antes, la institución dirigida por él era un espacio dedicado a la investigación clínica, el Hospital de Enfermedades de la Nutrición, creado en 1944. Sin embargo, existía otra institución que se encargaba de elaborar investigaciones sobre la alimentación de la población mexicana, y que llevaba el título de Instituto Nacional de Nutriología (INN), bajo la dirección del tercer médico recordado por Zubirán como señero de su formación, Francisco de Paula Miranda. Muchas veces el INN es confundido con el hospital de Zubirán, y se asume que es la misma institución que aparece en documentos de la década de 1940 como "Instituto de Nutriología" o simplemente como "Nutriología". En otras ocasiones es olvidado, opacado quizá por la amplia trayectoria de Zubirán y su hospital transformado en instituto. Sin embargo, el argumento central de este artículo no solo es recuperar parte de la historia de Nutriología y el proceso de su creación y posterior desaparición, sino mostrar que dicho instituto fue característico de un momento en la historia de la nutrición en el cual se enfatizó la exploración del "problema" de la mala alimentación como resultado de la pobreza de la población, y no solo como fruto de deficiencias específicas de nutrientes. ${ }^{2}$

Lo que muestro en este artículo es la génesis del Instituto Nacional de Nutriología, que explica en cierta medida por qué fue un espacio que reunió, desde su creación en 1943 hasta su cierre en 1956, un amplio grupo de investigadores quienes consolidaron una ambiciosa red de proyectos locales e internacionales sobre la alimentación en México. Uno de los objetivos que guían esta investigación es mostrar cómo se articularon los intereses de las fundaciones filantrópicas que financiaron al INN, con los intereses de los médicos encargados del estudio de la

\footnotetext{
2 Para referirme al Instituto Nacional de Nutriología, en este texto usaré el acrónimo INN, o Nutriología, como también se le conoció.
} 
alimentación local. El argumento es que más que pensar en una "dependencia" del INN hacia las instituciones internacionales, el Instituto funcionó como un organismo que, conociendo los intereses y proyectos internacionales, los articuló y empleó para promover sus propias líneas de investigación, siendo la principal el proyecto para conocer la alimentación de la población local. Gracias a ello, el equipo que trabajó en el INN mantuvo intercambios con varias instituciones nacionales e internacionales, y convirtieron al INN en un centro de investigación de punta en el terreno de la alimentación, con proyectos para conocer y mejorar la alimentación popular, urbana, rural e indígena, y con propuestas de cambios a la dieta local, todos bajo la perspectiva de la llamada "nutrición social", perspectiva que guiaba el trabajo de Miranda, en la cual, los problemas de la nutrición eran consecuencia de la pobreza.

La hipótesis que guía este trabajo es que, gracias al interés de Miranda y su acercamiento al problema de la nutrición desde una perspectiva poblacional, y como parte de un problema socioeconómico, se intentó mejorar la calidad de vida de los mexicanos a través de cambios en su dieta. Este acercamiento contrasta con los otros proyectos de investigación sobre nutrición, como el del Hospital de Enfermedades de Nutrición, donde se privilegió, en sus orígenes, la investigación de las carencias o excesos asociados con algunas enfermedades metabólicas, como la diabetes. La aproximación epidemiológica de Nutriología, cercana a lo perseguido por salud pública, se integró a otras estrategias sanitarias, como campañas de vacunación, creación de programas educativos, mejoramiento de servicios sanitarios como agua potable y drenaje, campañas de erradicación de enfermedades infecciosas, entre otras. La calidad de vida de la población, se argumentó, podía aumentarse al mejorar la alimentación, y para lograrlo el mayor obstáculo era el bajo salario, que impedía la compra de los alimentos sugeridos por los dictados de la nutrición. 
Los proyectos de alimentación con énfasis poblacional de Nutriología se encontraban en consonancia con la tendencia internacional de la nutrición social, misma que se interesaba por el estudio de las causas socioeconómicas de la mala alimentación, como veremos más adelante. Este enfoque pasó a segundo plano en las agendas de investigación en la segunda mitad del siglo xx, cuando se privilegió, en primer lugar, el aumento de la productividad agrícola para mejorar la distribución de alimentos, en segundo lugar, el análisis bioquímico de los alimentos, y finalmente, el estudio clínico de enfermedades asociadas con la alimentación.

Lo que intento mostrar es cómo estos cambios en los intereses de investigación, del estudio de poblaciones al énfasis en el aspecto clínico de la enfermedad, y del énfasis en el aspecto socioeconómico al análisis bioquímico, afectaron el financiamiento del INN, que no sobrevivió el cambio, y otras instituciones, como el Hospital de Enfermedades de la Nutrición, capitalizaron parte de lo hecho por el INN, y en ese proceso se eclipsó el trabajo previo de Nutriología y, con ello, su historia.

El presente texto se encuentra dividido en tres secciones. En primer lugar, se analiza el contexto en el cual surgió el Instituto Nacional de Nutriología, así como se explica por qué es parte de la tendencia de nutrición social de la época. En la segunda sección se analiza parte del trabajo realizado en dicho Instituto, y la última sección estudia el cierre de Nutriología y las condiciones nacionales e internacionales que lo enmarcaron.

\section{LOS PROYECTOS DE ALIMENTACIÓN \\ EN LA PRIMERA MITAD DEL SIGLO XX}

Diversas tradiciones han considerado la alimentación un elemento clave en el fomento de la salud de la población. Este aspecto ha sido poco estudiado por la historiografía, a pesar de que se menciona repetidas veces, tanto por los sujetos históricos 
como por los historiadores de la medicina, como origen de varios problemas de salud. A pesar de este reconocimiento del vínculo entre salud y alimentación, el estudio de las políticas públicas sanitarias se ha enfocado en los procesos infectocontagiosos y en las intervenciones sobre el espacio público para su higienización. ${ }^{3}$ La importancia de la alimentación como parte de la salud pública se hace evidente en muy diversas fuentes. Por ejemplo, a finales de la década de 1920, el Departamento de Salubridad Pública consideraba que la elevada mortalidad infantil se debía principalmente a dos factores: los “errores” de la alimentación y la falta de higiene. Los “errores” eran, de acuerdo con los médicos, los que realizaba la población en general al no saber cómo deberían comer de forma racional. Ante este diagnóstico, se crearon diversos programas de atención a estos problemas, enfatizando la divulgación de los conocimientos que consideraban necesarios. Entre estos programas destacan el de enfermeras visitadoras voluntarias de la Escuela de Salubridad, ${ }^{4}$ así como la creación de instituciones especializadas en el cuidado y atención de la alimentación infantil, como las denominadas Gotas de Leche. ${ }^{5}$ Además, se mantuvieron diversos programas de alimentación en instituciones de beneficencia. ${ }^{6}$ No es de extrañar que, en la década siguiente, durante la presidencia de Lázaro Cárdenas (1934 a 1940), la preocupación por procurar una dieta adecuada formara parte del plan sexenal para mantener

\footnotetext{
3 Véase por ejemplo, Rodríguez de Romo y Rodríguez Pérez, "Historia de la salud pública en México, siglos XIx y xx”, pp. 293-310; CARrillo, "Salud pública y poder en México durante el cardenismo, 1934-1940”, pp. 145-178; Magaña Valladares y Gudiño-Cejudo, Reseña bistórica de la Escuela de Salud Pública de México; y Cueto y Palmer, Medicine and Public Health in Latin America.

${ }^{4}$ Magaña Valladares y Gudiño-Cejudo, Reseña histórica de la Escuela de Salud Pública de México.

5 Viesca Treviño, “La Gota de Leche”, pp. 195-256.

6 Lorenzo Río, El Estado como benefactor.
} 
la salud del pueblo. ${ }^{7}$ Este interés propició que varios proyectos de alimentación quedaran parcialmente bajo la tutela del Departamento de Salubridad Pública, que coordinó esfuerzos con diferentes secretarías de Estado para conocer y "mejorar" la alimentación del pueblo mexicano.

Alimentar a una población urbana en crecimiento se entendía como un problema, pero lo que parecía más urgente era "mejorar" la alimentación de las poblaciones indígenas y campesinas. La dieta tradicional de estas poblaciones estaba basada en un consumo elevado de maíz, frijoles y pulque, y en menor medida de proteínas de origen animal, lo cual se asociaba con rasgos morales característicos del ser "indígena", como somnolencia, lentitud, pereza. Los pobres, quienes también consumían una dieta similar, personificaban también estos rasgos. A pesar de que se tenía un conocimiento general sobre la dieta tradicional, se consideraba necesario estudiar con mayor profundidad y rigor la alimentación de estos grupos. Los antropólogos realizaron diversos estudios en la población indígena, apoyados por médicos para estudiar la alimentación desde el punto de vista científico. Entre estas investigaciones podemos destacar los trabajos de Manuel Gamio a inicios de la década de 1920, quien, en su estudio La población del Valle de Teotibuacán colaboró con José Joaquín Izquierdo, en ese momento jefe de fisiología de la facultad de Medicina de la Universidad Nacional. Izquierdo llevó a cabo estudios fisiológicos y antropométricos, concluyendo que la alimentación indígena era deficiente. De manera similar, los estudios realizados por José Gómez Robleda sobre los tarascos, zapotecas y otomíes en la década de 1930, incorporaron los hallazgos realizados por médicos y biólogos sobre los hábitos alimenticios de la población indígena. Sus conclusiones reiteraron lo que Gamio había encontrado, que existía una deficiencia

7 Carrillo, "Salud pública y poder en México durante el Cardenismo, 1934$1940 ”$, p. 149. 
en el consumo de proteínas de origen animal, carencia que se relacionaba con el carácter "primitivo" de estas poblaciones.

Otro proyecto para el estudio de la alimentación indígena fue coordinado por Lucio Mendieta y Núñez en el Instituto de Investigaciones Sociales de la Universidad Nacional. Ahí se creó una sección de investigaciones alimentarias, que al parecer tuvo una vida efímera. Hay que hacer una aclaración sobre los resultados obtenidos en estos proyectos antropológicos. En estas investigaciones y sus resultados se incorporaba la visión racializada que tenían estos investigadores. Es decir, ellos consideraban que la alimentación "normal” consistía en un elevado consumo de proteína de origen animal, noción que era validado por estudios fisiológicos hechos en Europa y Estados Unidos, donde su dieta había sido el modelo, el "estándar". De esta manera, al estudiar un grupo étnico diferente, considerado en ese momento "primitivo", su alimentación, diferente a la "normal”, adquiría por analogía esta característica de "anormal”, que se traducía en "primitiva”, consolidando el prejuicio racial existente en la época. Este tipo de sesgos en la investigación sobre la alimentación se mantuvo hasta bien entrado el siglo xx, y las investigaciones antropológicas y nutriológicas del periodo que se discute en este artículo compartieron la noción de una dieta "modelo" contra otra desconocida, diferente, anormal y que se interpretó como "inferior". ${ }^{8}$ La dieta indígena, campesina o de los pobres, era diferente y, por lo tanto, inferior.

Durante el cardenismo se intentaron consolidar en un solo sitio los proyectos relacionados con el estudio de la alimentación

\footnotetext{
8 Aguirre Beltrán, Antropología médica, pp. 163-170. Habría que analizar con mayor detalle cada investigación en particular para realizar un diagnóstico más adecuado; sin embargo, esta línea argumentativa se mantiene en varios proyectos de investigación del periodo, no solo en México sino también en otros países. Sobre la incorporación de las ideas de una alimentación primitiva y el sesgo racista de los investigadores, véase VARGas-Domínguez, "The 'problematic' Otomi” y VArgas-Domínguez, "El metabolismo racial”.
} 
y la creación de la Oficina General de Higiene de la Alimentación, dividida en tres secciones: Investigación de la Alimentación Popular, Higiene Veterinaria y Registro. La sección de Alimentación Popular fue la más importante y gestionó la creación de una comisión con miembros de varias dependencias. Como jefe de esta sección estuvo Jesús Díaz Barriga, de la Secretaría de Salubridad, quien, junto con el sociólogo Siegfried Askinasy, intentaron conocer el estado nutricional de la población pero, de acuerdo con Gonzalo Aguirre Beltrán, dicha sección no tuvo resultados importantes. Sin embargo, de ahí surgió la comisión que a la postre sería la más relevante por sus alcances y las redes construidas a su alrededor, la Comisión Nacional de Alimentación (CNA), creada por decreto el 13 de marzo de $1936 .{ }^{9} \mathrm{La}$ CNA fue encabezada por un representante del Departamento de Salubridad Pública, siendo su primer director el médico José Quintín Olascoaga. La importancia de la CNA, al menos en el papel, era evidente por la diversidad y número de miembros, que eran representantes de las Secretarías de Agricultura, Comunicaciones y Obras Públicas, Hacienda y Crédito Público, Economía Nacional, Educación Pública, y de los Departamentos Agrario, de Asistencia Infantil, de Prensa y Propaganda, de Asuntos Indígenas, del Trabajo, del Departamento Forestal y de Caza y Pesca, así como representantes de la Beneficencia Pública y del Consejo Nacional de Educación Superior e Investigación Científica. ${ }^{10}$ De los miembros, seis eran médicos, lo cual reforzó la mirada medicalizada sobre la forma de enfrentar el problema de la alimentación en el país. En todos estos proyectos se asociaba el carácter "primitivo" o "retrasado" de las poblaciones pobres, campesinas o indígenas con una alimentación insuficiente, con

\footnotetext{
9 Aguirre Beltrán, Antropología médica, pp. 167-168; Quintín OlascoaGA, Nutriología, t. 1, pp. 20-21.

10 "Reglamento General de la Comisión Nacional de la Alimentación", ca. 1938, AGN, SEP, DPH, C 35472, E 17-14-5-68; Payne a Ferrell, 6 de febrero de 1942, RAC, RFR, RG 1.1, S 323, B 12, F 80.
} 
pocos alimentos de origen animal, idea que fue reiterada por los antropólogos y médicos que trataron de enfrentarse al "problema de la nutrición" y que les permitía, al modificar la dieta, cambiar a la población.

La CNA tuvo como objetivo estudiar los "problemas mexicanos de nutrición humana" con tres enfoques principales: higiénico, económico y educativo. Entre sus metas se encontraba aumentar la producción nacional de "sustancias alimenticias", que debe interpretarse como buscar las vías para incrementar de la producción y el consumo de proteínas de origen animal, con el fin de "obtener una ración nutritiva equilibrada, suficiente y sana" para los mexicanos. Lo "sano" se relacionaba con un consumo elevado de proteínas de origen animal, o "alimentos protectores” como les llamaban. ${ }^{11}$ Para lograr la ración adecuada y que estuviera al alcance de la población, se requería que se redujeran los costos de los alimentos con mecanismos como el aumento de producción, el control de precios y la mejoría de los canales de distribución.

Desde la CNA se realizaron estudios sobre dietología "normal y terapéutica" y a partir de ellos se elaboraron unos cuadros básicos de alimentación para su uso en algunos hospitales, guarderías y escuelas, principalmente en la ciudad de México. La Comisión presumía que sus trabajos habían servido para

[...] preparar el ambiente que era indispensable para realizar, en forma amplia y eficiente, los trabajos encaminados para que el pueblo mexicano, en su totalidad, llegue a disponer de alimentación adecuada a sus necesidades y en concordancia con las adquisiciones de la ciencia de la nutriología y las demás ciencias sociales. ${ }^{12}$

11 "Reglamento General de la Comisión Nacional de la Alimentación”, ca. 1938, AGN, SEP, DPH, C 35472, E 17-14-5-68.

12 Comisión Nacional de la Alimentación, "Organización, funciones y actividades, octubre de 1941”, AHSSA, SSA, SubSyA, c. 17, exp. 11, f. 41; 
La llamada "ciencia de la nutriología" se encontraba así en el centro del proyecto cardenista de alimentación y transitaba entre el campo de la salud pública y la fisiología de la alimentación.

La Secretaría de Asistencia Pública (SAP), creada en 1937 en una reestructuración de las dependencias del Estado, ${ }^{13}$ tomó a la cNA bajo su control en 1938, aunque se mantuvo fuertemente vinculada con Salubridad, al seguir siendo el representante de Salubridad el jefe de la Comisión. Esta incorporación de la CNA a la Asistencia Pública tuvo como finalidad establecer criterios "dietéticos" de la alimentación que se ofrecía en los establecimientos de dicha Secretaría. ${ }^{14}$ Este objetivo fue rápidamente rebasado y la CNA se propuso otro aún más ambicioso: estudiar la alimentación popular de todo el país. Cabe destacar que los médicos relacionados con los proyectos de alimentación de la Comisión Nacional de Alimentación, como José Quintín Olascoaga o Jesús Díaz Barriga, presentaron trabajos en el Primer Congreso Indigenista Interamericano en Pátzcuaro, Michoacán, en 1940. En sus presentaciones reiteraban la idea de que la alimentación indígena debía cambiar, pero que requería ser estudiada. En las resoluciones de dicho congreso, se sugirió la creación de comedores en "las regiones más densamente pobladas por elemento laborista”. Asimismo, se sugería que cada país creara una "Comisión coordinadora de las actividades de las diversas dependencias oficiales" encargada de "formular los programas adecuados de producción y distribución de alimentos" entre las comunidades rurales e indígenas..$^{15}$ Estos

\footnotetext{
"Reglamento General de la Comisión Nacional de la Alimentación”, ca. 1938, en AGN, SEP, DPH, c. 35472, exp. 17-14-5-68.

13 Rodríguez de Romo y Rodríguez Pérez, "Historia de la salud pública en México: siglos XIX y xx”.

${ }^{14}$ Segura Millán, "Informe sintético de las labores desarrolladas hasta la fecha por la Comisión de Alimentación”, 1941, AHSSA, BP, D, SS, C16, E2.

15 "El Primer Congreso Indigenista Interamericano", en Boletín Bibliográfico de Antropología Americana (1937-1948), 4: 1 (1940), pp. 15-17.
} 
proyectos cristalizarían a lo largo de la década de 1940, y sus encargados fueron figuras importantes en el panorama de la nutrición en México, como José Quintín Olascoaga, Salvador Zubirán y Francisco de Paula Miranda.

Quintín Olascoaga, primer presidente de la CNA, siguió las propuestas sociales del nutriólogo argentino Pedro Escudero, que se vieron reflejadas en las publicaciones que hizo posteriormente. A finales de la década de 1930 e inicios de la siguiente, Quintín Olascoaga realizó una estancia de investigación en Buenos Aires, en el Instituto de Nutrición de Pedro Escudero, quien proponía el estudio de la nutrición como parte de un problema social más amplio, y cuyas raíces eran primordialmente económicas, más que vinculadas con alguna tara social o biológica. Esta impronta quedaría en Quintín Olascoaga y en su trabajo posterior en el INN, como veremos más adelante. Los archivos revelan que Francisco de Paula Miranda lo sustituyó en la presidencia de la CNA hacia 1938, cuando Miranda empezó a aparecer signando como presidente de la Comisión. La elección de Miranda como cabeza de la CNA no es de extrañarse dado que ya era un personaje reconocido dentro del gremio médico y había sido miembro de la Academia Nacional de Medicina (ANM) desde 1923, y fue su presidente entre 1934 y $1935 .{ }^{16}$

Miranda en la CNA mantuvo el objetivo de resolver el problema de la alimentación al mejorar las condiciones de vida de la población, lo cual no era un planteamiento excepcional

16 De hecho, es un tropo común en las investigaciones históricas insistir en que Quintín Olascoaga fue presidente de la CNA, reiteración que parece provenir de un texto que publicó el mismo Olascoaga en 1946 bajo el título de Nutriología. En los documentos del Archivo Histórico de la Secretaría de Salud, Miranda firmaba como presidente desde 1938, pero en algunos folletos publicados por la CNA, Olascoaga aparece como presidente aún en 1941. Una investigación más amplia se está llevando a cabo sobre Quintín Olascoaga y su relación con la nutrición en Argentina y el proyecto de nutrición social de Pedro Escudero. Sobre el trabajo de Escudero, véase Buschini, "La alimentación como problema científico". 
de México. Médicos, nutriólogos y políticos en el ámbito internacional pensaban que la "alimentación imperfecta" de las "clases trabajadoras" podía llevar a la desobediencia civil e incluso a generar más guerras. Miranda sostenía que era necesario cubrir no solo una dieta mínima, sino una dieta óptima. La dieta mínima solo satisfacía los requerimientos mínimos, pero una dieta óptima tenía como objetivo proporcionar una alimentación adecuada no solo en cantidad sino en calidad. Este enfoque fue uno de los argumentos centrales de la nutrición social y una guía durante toda la vida de Miranda: preocuparse por las perspectivas sociales, en un terreno más cercano a la salud pública, y no solo por los problemas individuales, más cercanos a la práctica clínica. ${ }^{17}$

La sensación de urgencia ante la posibilidad de un conflicto social generado por la mala alimentación propició que, a mediados de la década de 1930, diversos grupos de expertos en alimentación se incorporaran a organismos internacionales, como la Sociedad de Naciones, la Oficina Internacional del Trabajo, el Instituto Internacional de Agricultura de Roma y la Fundación Rockefeller (FR). Fueron ellos quienes, en conjunto, impulsaron el desarrollo de más y mejores investigaciones relacionadas con la nutrición para evitar este problema. ${ }^{18}$

El resultado de estos esfuerzos internacionales fue la compilación de las más novedosas investigaciones sobre el tema, en un reporte publicado en 1936 titulado "The Problem of Nutrition" en el cual se sentaban los lineamientos nutricionales que debían seguir por todas las naciones miembros de estos organismos. ${ }^{19}$ Estos lineamientos establecían que había una relación directa entre los requerimientos nutricionales, la pobreza y la enfermedad, y proponían la creación de centros locales de investigación

17 Smith, "Nutrition Science and the Two World Wars", p. 150.

18 Barona, "Nutrition and Health. The International Context During the Interwar Crisis”, p. 88.

19 Barona, The Problem of Nutrition. 
especializados en nutrición para consolidar la información existente sobre la alimentación de distintas naciones. El documento fue considerado como una guía a seguir para los proponentes de la nutrición social. La nutrición social, de acuerdo con James Vernon, fue un movimiento que adquirió fuerza a partir de la década de 1920, el cual proponía que la investigación sobre alimentación estuviera "atenta a los significados sociales de la comida, y a la pobreza como la causa de la malnutrición”. ${ }^{20} \mathrm{Se}$ entendía que se debían atacar las causas socioeconómicas de la mala alimentación dado que, argumentaban, no era suficiente la investigación clínica sobre enfermedades carenciales o por exceso, ni tampoco bastaba el análisis de los alimentos.

La nutrición social podemos considerarla como un conglomerado de prácticas sociales, médicas y científicas que se emplearon para atacar el problema de la nutrición, y que asumía que la mala alimentación tenía un origen socioeconómico y debía interpretarse desde una perspectiva poblacional e integral. En este sentido, la nutrición social se encontraba muy cerca de los preceptos de la medicina social. Desde el final de la Revolución se consideró que el Estado era el responsable de la salud de la población, elevando este derecho a un rango constitucional. Esta responsabilidad podemos rastrearla hasta el porfiriato, cuando la alimentación, estudiada por el higienismo, se incorporó posteriormente como parte de las mejoras a la salud recomendadas por el gobierno revolucionario. La alimentación se sumaba a las prácticas de prevención impulsadas por la medicina social, considerándola una de las primeras barreras para la contención de epidemias y como herramienta que permitía modificar taras hereditarias, como una estrategia eugenésica. De acuerdo con Claudia Agostoni, los proyectos de medicina social se enfocaron a las poblaciones campesinas e indígenas, siendo las receptoras de las intervenciones masivas "hasta el corazón de

20 Vernon, Hunger. A Modern History, pp. 119-120. 
las clases populares". ${ }^{21}$ Podemos argumentar que la nutrición formaba parte de las políticas de la medicina social, pero que su papel ha sido poco estudiado, lo mismo que sus actores, como Quintín Olascoaga, Miranda o Zubirán.

\section{DE LA COMISIÓN NACIONAL DE ALIMENTACIÓN}

AL INSTITUTO NACIONAL DE NUTRIOLOGÍA

Miranda, director de la CNA, junto con su jefe inmediato, Salvador Zubirán, cabeza de la Secretaría de Asistencia Pública, gestionaron y lograron la instalación de un Comedor Nacional Familiar en 1941, que fue conocido como Unión Nacional, del cual Miranda fue nombrado director. ${ }^{22} \mathrm{El}$ Comedor fue excepcional porque se pensó como un laboratorio de estudios sobre alimentación y como un modelo para la creación de futuros comedores a nivel nacional, es decir, no cumpliría solo los papeles tradicionales de otros comedores, herederos del asistencialismo privado o público, sino que sería un sitio de producción de conocimientos sociales y científicos.

El Comedor Nacional Experimental "Unión Nacional” tuvo como sede un anexo del mercado Abelardo L. Rodríguez, en el centro de la ciudad de México. Situar el Comedor ahí obedecía a que dicha zona era considerada de corte popular, con habitantes que cubrían el perfil requerido para ser asistidos y estudiados. Los asistentes al Comedor eran pobres, obreros y burócratas con bajos salarios, quienes debían servir como modelo para evaluar el impacto en la salud de un proyecto de alimentación

\footnotetext{
${ }^{21}$ Como llama Rodríguez Ocaña (citado por Agostoni) a la forma en que se intervino en las clases populares desde la medicina en los proyectos de salud pública de la primera mitad del siglo xx. Para el análisis de los proyectos médicos sociales en México véase Agostoni, "Médicos rurales y medicina social en el México posrevolucionario (1920-1940)".

22 "La obra de los Comedores Nacionales", 1941, AHSSA, BP, D, SS, C 16, E 5 .
} 
basado en la ciencia, "mejor" que lo que consumían habitualmente. El mercado, desde su inauguración en 1934, se había considerado el "más grande y moderno" no solo de la ciudad sino de América Latina, y la instalación del Comedor mantenía el espíritu de pertenecer a este proyecto "modernizador" de gran envergadura. ${ }^{23} \mathrm{El}$ Comedor tuvo una excelente acogida y, debido a la amplia demanda de los desayunos escolares y familiares, se planeó enseguida un segundo Comedor, también pensado como laboratorio. En este nuevo proyecto, además, se planeó la creación de un instituto especializado en nutrición que debía funcionar anexo al mismo, para dirigir las labores de investigación de una manera directa. ${ }^{24}$

Como se ha mostrado en otra investigación, para la creación de este nuevo instituto, Miranda estableció una red que incorporó a los representantes de la International Health Division (IHD) de la FR, que funcionaba en México a través de la Oficina de Especialización Sanitaria (OEs). ${ }^{25}$ La FR ofreció para la construcción del Instituto la suma de 20000 dólares en $1941,{ }^{26}$ y bajo este auspicio, Miranda y un grupo de médicos e investigadores le enviaron una misiva al presidente Manuel Ávila Camacho, en la cual se hacía hincapié en la suma prometida por la FR y resaltaban el interés particular del vicepresidente estadounidense, Henry A. Wallace, en la creación de un instituto especializado en alimentación en el país. Previamente Wallace, un gran promotor de la agricultura, había insistido ante la junta directiva de la FR que era necesario estudiar la producción de alimentos en México

23 "La obra de los Comedores Nacionales", 1941, AHSSA, BP, D, SS, C 16, E 5. Sobre las prácticas realizadas en los comedores, véase Aguilar RodríGUEZ, "Cooking Modernity", pp. 177-205.

24 "Construcción del Comedor Nacional Experimental 'Unión Nacional”, 1941, AHSSA, $B P, D, S S$, C 16, E 5; Zubirán, "Informes de la Secretaría de Estudios para el Informe Presidencial”, 1942, AHSSA, BP, D, SS, C 22, E 10. ${ }^{25}$ Vargas-Domínguez, "La construcción del Instituto Nacional de Nutriología en México".

26 RAC, $R F R$, RG 1.1, S 323, B 12, F 80. 
y que no había mejor institución para iniciarlo que la misma Fundación, dada su fuerte presencia en nuestro país y sus relaciones con el sistema de salud local. El interés en la agricultura sugerido por Wallace resultaría crucial en el futuro del INN. ${ }^{27}$

En la carta que le enviaron a Ávila Camacho, firmada por la Sociedad Mexicana de Nutriología y cuyo presidente era Miranda, se explicaban las ventajas económicas que suponía la creación de un instituto de nutrición para México, dado que, en el periodo de la posguerra, México era considerado un país productor de insumos básicos indispensables para la reconstrucción de Europa:

Es evidente, de una parte, la importancia que las funciones del nuevo organismo [el Instituto] tendrán en la determinación de una correcta política económica, especialmente en lo que hace a la producción agrícola, y de la otra, resulta imperioso tener a la vista este proyecto en el momento en que se elabora el Presupuesto General de Egresos para 1942. ${ }^{28}$

No fue en 1942, pero sí en 1943 cuando, anexo al Comedor, y gracias a las negociaciones de Miranda, iniciaron las labores del primer instituto en México especializado en nutrición, y que recibió el nombre de Instituto Nacional de Nutriología,

27 Procel, "Memorandum que la profesora Áurea Procel presenta al C. Presidente de la República, acerca del establecimiento en México, por cooperación internacional, de un instituto encargado de investigar lo relativo a la alimentación popular”. AGN, $P, M A C, 103202$, (523.1/24-523.1/73). El Programa Agrícola Mexicano sería el origen de lo que posteriormente sería conocido como la revolución verde. Birn, Marriage of convenience; Cueto, Missionaries of Science. Sobre la revolución verde, véase Cullather, The Hungry World.

28 Áurea Procel, "Memorandum que la profesora Áurea Procel presenta al C. Presidente de la República, acerca del establecimiento en México, por cooperación internacional, de un instituto encargado de investigar lo relativo a la alimentación popular”, 1941. AGN, $P, M A C, 103202$ (523.1/24-523.1/73). 
fusionándose con la CNA, que desapareció. Miranda quedó a la cabeza de éste y una nueva etapa de estudios sobre nutrición se inició en México.

El Instituto Nacional de Nutriología empezó a funcionar bajo la Dirección General de Investigación Científica y Técnica del Departamento de Salubridad. En Nutriología se consolidó un grupo de investigadores interesados en la nutrición y la fisiología de la alimentación, siendo la mayoría médicos y con vínculos con la medicina estadounidense, entre ellos Guadalupe Eguiluz, José Calvo de la Torre, Omar Cravioto, René O. Cravioto, Guillermo Massieu, Dolores Salazar, Flor de María Figueroa, José Quintín Olascoaga, Jesús Díaz Barriga, Alfredo Ramos Espinoza y Rafael Segura Millán, entre otros. Parte de los intereses de la CNA se mantuvieron presentes en el nuevo instituto, en especial la necesidad de investigar las causas de la mala alimentación desde la perspectiva de la nutrición social. Se mantuvo la idea de que no se conocía adecuadamente la alimentación popular. El Instituto inició funciones con las secciones de Estudios Económicos y Dietarios, la de Estudios Antropométricos, la sección de Bromatología, la de Estudios Clínicos y Fisiológicos, la sección de Coordinación y finalmente la sección de Acción Social y Divulgación. Buena parte de la investigación sobre alimentación popular urbana se llevó a cabo en los Comedores 1 y 2 que había creado la CNA y que seguían vinculados con Nutriología. Sin embargo, era necesario que se realizara mayor investigación sobre la alimentación de las poblaciones rurales e indígenas para ofrecer un diagnóstico claro de la situación general. Los investigadores del INN colaboraron con antropólogos en los proyectos de estudio de la alimentación indígena, consolidando investigaciones que se integraron a lo que aún se considera investigaciones ejemplares de la antropología médica mexicana. Una de las herramientas que se empleó fue el levantamiento de encuestas de alimentación. Las encuestas no solo permitían conocer los hábitos de alimentación de la población o el estado 
de salud de los encuestados, sino que también indagaban sobre las características socioeconómicas de los sujetos de investigación. Los expertos coincidían con la necesidad de las encuestas para evaluar los efectos de la "mala alimentación popular" en la salud. Conocer estos datos ofrecería la posibilidad de proponer mejoras a su alimentación, fuese por medio de la educación o, una vez reconocidas las deficiencias en la alimentación, orientar la producción agrícola para subsanarlas. ${ }^{29}$

Miranda se había comprometido a dar prioridad a las investigaciones que la FR considerara necesarias, a cambio del financiamiento inicial del Instituto. El mismo gobierno estadounidense cooperó directamente mediante la Coordinación de Asuntos Interamericanos, que en 1943, le dio al Instituto 90000 pesos. La presencia de estas fuertes sumas de dinero (en contexto, la misma oficina había otorgado al gobierno mexicano 500000 pesos para la campaña contra la oncocercosis) parecieran indicar que los proyectos del Instituto fueron dirigidos desde "fuera". Mi propuesta es que no fue así necesariamente. Los investigadores mexicanos, al menos en los inicios del INN, compartían los mismos intereses de investigación que los organismos internacionales, lo cual motivó más una colaboración que un sometimiento a los deseos foráneos. Además, varios miembros del INN fueron entrenados en Estados Unidos, lo cual explica en cierta medida estos intereses compartidos. Posteriormente, con el cambio de aproximaciones al estudio de la nutrición y el énfasis agrícola que se dio desde mediados de la década de 1940, sí hubo un cambio en el apoyo y el INN vio mermada su capacidad de investigación, pero inicialmente, parafraseando a Anne-Emanuelle Birn, el INN funcionó como un "matrimonio por conveniencia" de diversos intereses, locales e internacionales. Para lograrlo,

29 Aguirre Beltrán, Antropología médica, p. 169; Departamento de SALubridad Pública, Memoria Anual del Departamento de Salubridad Pública correspondiente al periodo 1 septiembre 1942-31 agosto 1943, pp. 168-170. 
Miranda y George C. Payne, representante de la IHD en México, mantuvieron una estrecha colaboración en el estudio de la alimentación popular. ${ }^{30}$

El INN colaboró con dos divisiones de la FR. En primer lugar, la IHD a través de la OES, se encargó de la investigación concerniente a la alimentación popular. En segundo lugar, la Natural Sciences Division (NSD) creó una Oficina de Estudios Especiales (OEE) en México vinculada con la Secretaría de Agricultura, misma que llevó a cabo desarrollos e investigaciones sobre métodos agrícolas modernos. La oeE fue la encargada de lo que se conoció como Programa Agrícola Mexicano (PAM) y estuvo dirigida en sus primeros años de funcionamiento por J. George Harrar, quien más tarde sería presidente de la FR.

Las dos divisiones de la FR, a pesar de compartir el objetivo de buscar medios para solucionar el problema de la nutrición del pueblo mexicano, ofrecían caminos distintos para lograrlo. Como ya he mencionado, la IHD-OEs buscaba conocer primero a la población y sus requerimientos específicos. Su punto de partida eran las encuestas para conocer el estado nutricional y el consumo local y, a partir de ellas, tratar de hacer intervenciones en la alimentación, tomando siempre en cuenta el aspecto socioeconómico de la población atendida, aproximación semejante a la de los integrantes del INN. Por su parte, la NSD-OEE asumía que el medio por medio del cual se podía atacar el problema de la nutrición era el aumento en la producción agrícola local de los cereales de alto consumo, como maíz, trigo y soya. ${ }^{31}$ En este sentido, el PAM apuntaba a la modernización de técnicas de cultivo locales y a la búsqueda de variedades de cereales de alto

30 Departamento de Salubridad Pública, Memoria Anual del Departamento de Salubridad Pública correspondiente al periodo 1 septiembre 1942-31 agosto 1943, pp. 15-16.

${ }^{31}$ Downs a Harrar, April 19, 1948, AHSSA, SSA, SubSyA, C 10, E 4; Cobb, "The historical backgrounds of the Mexican Agricultural Program", RAC, RFR, $R G$ 1.2, S 323, B 9, F 62. 
rendimiento productivo. En teoría, el aumento en la producción y una mejor distribución se traducirían en la disminución de costos, haciendo estos alimentos más accesibles a las poblaciones necesitadas.

Miranda, en lo personal, se encontraba más cercano a los intereses de la IHD y de Payne que a los intereses de la NSD con Harrar. La nutrición social privilegiaba la formulación de encuestas para conocer qué era lo que se necesitaba, más que asumir una deficiencia inicial que podría solventarse con la búsqueda de híbridos agrícolas de alta productividad. La colaboración del INN con la IHD-OEs se basó en la realización de diversas encuestas entre los atendidos en los Comedores en la ciudad de México, así como en encuestas de nutrición en áreas rurales, como las realizadas en Guanajuato, en Capula en Michoacán, así como el estudio de los otomíes del Valle del Mezquital en Hidalgo, como veremos más adelante. ${ }^{32}$ En paralelo a las encuestas, se recolectaron muestras de alimentos tradicionales que debían analizarse en el INN para conocer su composición. Estos estudios bioquímicos de los alimentos también se pudieron hacer gracias al interés de la FR en el análisis de alimentos y a su patrocinio para la compra de instrumentos en el INN.

La colaboración del INN con la FR se amplió cuando la Oficina Sanitaria Panamericana consideró que el instituto era el lugar ideal para el estudio de la nutrición mexicana. Esto generó la creación de un laboratorio que fue financiado por la Fundación Kellogg (FK), la IHD y la NSD de la FR para el estudio de los alimentos mexicanos. ${ }^{33}$ Este laboratorio también sirvió para evaluar la composición de los granos producidos por el PAM:

\footnotetext{
32 Anderson, Calvo de la Torre et al., "Nutrition appraisals in Mexico", pp. 1126-1135. La revista Nutriología es esquiva. A pesar de que se le menciona en diferentes ocasiones, no hay muchos ejemplares disponibles de dicha revista; al parecer algunos están resguardados en la Universidad Pedagógica Nacional y otros en el AHSSA.

33 RAC, RFR, RG 1.1, S 323, B 12, F 81.
} 
los granos eran producidos por la NSD-OEE y el análisis de los granos lo hacía Nutriología. El laboratorio se instaló inicialmente en el edificio de la Escuela de Salubridad e Higiene, y la capacitación del personal fue en parte pagada por la IHD. ${ }^{34}$ Esta colaboración produjo las tablas de composición de alimentos locales más completas hasta ese momento, además de la publicación de investigaciones sobre la calidad de los alimentos locales como la tortilla. ${ }^{35}$

El proyecto del INN, como parte de la necesidad de educar a la población para que consumiera "racionalmente", incluyó la impartición de cursos de dietética, además de la formación de cuadros de personal especializado, en un programa similar al de las enfermeras visitadoras, pero, en este caso, dedicado al levantamiento de encuestas de alimentación. Los cursos de Dietética estuvieron bajo la dirección de José Quintín Olascoaga, quien mantenía la visión de nutrición social de De Paula Miranda, complementada con la influencia de las enseñanzas de la nutrición social del nutriólogo Pedro Escudero, con quien había hecho una estancia en Buenos Aires. Estos cursos fueron impartidos en el Hospital de Cardiología, como parte de las labores de formación de personal del INN. ${ }^{36}$

Los datos de las primeras encuestas de nutrición, elaboradas en colaboración con William D. Robinson de la IHD, fueron

\footnotetext{
34 RAC, RFR, RG 1.1, S 323, B 12, F 79.

35 De entre las publicaciones más reconocidas, en las cuales participó directamente el inn, destacan: Cravioto, Lockhart, Anderson, De Paula Miranda, Harris, Aguilar, Tapia, et al., "Composition of Typical Mexican Foods”, pp. 317-329; Cravioto, Anderson, Lockhart, De Paula MiranDA y HARris “Nutritive Value of the Mexican Tortilla”, pp. 91-93; Cravioto, Massieu, Guzmán y Calvo de la Torre, “Composición de alimentos mexicanos”, pp. 129-155.

${ }^{36} \mathrm{La}$ influencia de Escudero en Quintín Olascoaga puede verse en su texto con el cual dictaba cursos. Hay una investigación en proceso sobre esta relación entre la enseñanza de la nutrición argentina y la mexicana. QuINTín Olascoaga, Nutriología.
} 
presentados por De Paula Miranda en foros internacionales, como la reunión de la Conferencia de las Naciones Unidas sobre Agricultura y Alimentación de 1943, y que dio pie a la Organización para la Agricultura y la Alimentación de las Naciones Unidas (FAO). En ella, De Paula Miranda se posicionó como uno de los expertos mundiales en nutrición, como un científicodiplomático que participó como experto y como representante de México en múltiples foros. El INN incluso publicó su propia revista de divulgación de sus investigaciones, de corta vida, titulada Nutriología a partir de 1951. De esta manera, el estudio de la alimentación en México se ubicaba a la cabeza de las investigaciones sobre el tema en Latinoamérica. ${ }^{37}$

Uno de los proyectos donde más se notó el influjo de la nutrición social en el INN fue el estudio sobre los indígenas otomíes habitantes del Valle del Mezquital, situado a una centena de kilómetros de la ciudad de México. Esta investigación fortaleció los vínculos de investigadores del INN, como José Calvo y Gloria Serrano, con investigadores de la FR, principalmente George C. Payne y Richmond K. Anderson. Estos estudios en el Valle del Mezquital fueron la continuación de un programa de investigación y acción social creado por el gobierno mexicano a mediados de la década de 1930. Los otomíes se convirtieron en una "población del conocimiento", una población en la cual la producción de conocimiento estaba distribuida entre los investigadores y los sujetos estudiados, y que sumaba nuevos significados a las categorías y clasificaciones impuestas por la ciencia a estos mismos sujetos, en un "efecto bucle" de retroalimentación positiva tanto para los otomíes como para los investigadores..$^{38}$

\footnotetext{
37 Sobre la participación de De Paula Miranda en dicha conferencia, pueden verse VARgas-Domínguez, "Entre la nación y el mundo: la nutrición en México en la Conferencia de las Naciones Unidas sobre alimentación de 1943” y VARgas-Domínguez y Minor García, "Mexican Scientists in the Making of Nutritional and Nuclear Diplomacy", pp. 34-56.

38 Vargas-Domínguez, "The 'problematic' Otomi”.
} 
Los comedores experimentales de la ciudad de México, en cierta manera, intentaron reproducir las formas de intervención que se habían realizado en el Mezquital, pero ahora en una población urbana que, lo mismo que los otomíes, era considerada marginal. Cambiar la alimentación se consolidó como el proyecto sugerido por los médicos para mejorar las condiciones de vida de estas poblaciones, sugerencia que se mantuvo entre los antropólogos en el auge del indigenismo.

El estudio de la población otomí fue retomado en 1949 por el encargado de la sección de nutrición de la Oficina Sanitaria Panamericana (OSP), Nevin S. Scrimshaw, quien, además de elogiar los avances en el estudio de la composición de alimentos en México -efectuados por el INN-, reconocía que el estudio de la alimentación otomí había descubierto que en su dieta suplían la deficiencia de ácido ascórbico, escasamente presente en los alimentos que consumían, con la ingesta continua de pulque. De esta manera el pulque debía considerarse crucial en la dieta local, a pesar de las restricciones morales existentes sobre el consumo de esta bebida alcohólica. Este tipo de datos, de acuerdo con Scrimshaw, eran la muestra de la necesidad de impulsar más investigaciones para desarrollar programas locales de alimentación, dado que "intentar imponer arbitrariamente dietas estadounidenses o europeas, con el fin de mejorar la nutrición, constituiría probablemente un fracaso, puesto que económica y culturalmente resultaría inaceptable, a pesar de que en teoría resulte deseable desde el punto de vista de la alimentación". ${ }^{39}$ Las encuestas demostraban así su utilidad al considerar las necesidades de las poblaciones locales, más que apuntar a una solución universal.

Para 1946, el trabajo que hacía el INN era conocido a nivel mundial. La Fundación Kellogg consideraba que México y

39 SCRImshaw, "Estudios sobre los problemas de la nutrición en la América Latina”, pp. 1201-1202. 
el INN debían ser el modelo para un nuevo centro de investigación a crearse en Centroamérica bajo la tutela de la osp y con financiamiento de ambas instituciones. Se esperaba que México fuese un "área de demostración en nutrición para el resto de Latinoamérica", y que los integrantes del planeado instituto hicieran estancias en México para aprender cómo funcionaba el INN y cómo llevaba a cabo estudios sobre alimentación popular. ${ }^{40}$

El proyectado centro se creó en 1949 bajo el nombre de Instituto de Nutrición de Centro América y Panamá (INCAP), y su primer director fue Scrimshaw, quien reconoció la importancia de las investigaciones del INN y las tomó como modelo para el INCAP, institución que posteriormente se convertiría en uno de los referentes en estudios nutricionales en Latinoamérica. La financiación del INCAP provino de los gobiernos centroamericanos, pero una buena parte fue otorgada por la OsP y la FK. La creación de este instituto resultó crucial en el ocaso del INN.

EL DECLIVE DEL ENFOQUE SOCIAL Y EL CIERRE DEL INN

La evaluación de la nutrición de la población era una tarea compleja que requirió que el INN la fragmentara en pequeñas parcelas de investigación. Por un lado, las encuestas de nutrición de la población de la ciudad de México, iniciadas en colaboración con Wilbur D. Robinson de la IHD en los Comedores, continuaron bajo la dirección del INN. Robinson fue el encargado de evaluar el efecto de los cambios en las dietas de los asistidos en los Comedores, mensurable gracias a encuestas. Posteriormente, Richmond K. Anderson se convirtió en el nuevo contacto de la IHD, lo cual se conjugó con el inicio de operaciones del INN en 1943. Anderson tenía la consigna de hacer encuestas de alimentación ya no a la población en general, lo que hasta ese

40 “The central American Nutrition Institute", 1946, RAC, RFR, RG 6.13, S1.1, B 8, F 94. 
momento se había hecho en los Comedores, sino que el énfasis debía ponerse en grupos poblacionales que presentasen desnutrición severa, por lo que sus estudios, siempre en colaboración con De Paula Miranda, fueron orientados hacia la evaluación de las poblaciones rurales y la búsqueda de deficiencias graves, que requirieran una participación más activa del personal médico para mejorar sus condiciones. El estudio de los otomíes fue un ejemplo de este interés, dada su precaria condición socioeconómica y que eran considerados uno de los grupos más pobres del país. ${ }^{41}$

John A. Ferrel, director asociado de la IHD, ya había advertido a su representante en México, el Dr. Payne, que se debían investigar los aspectos clínicos de la alimentación. Puesto que se habían hecho encuestas en poblaciones "relativamente prósperas", mismas que no habían mostrado "la cantidad de deficiencias nutricionales que esperábamos encontrar”, se requería encontrar en el país casos clínicos de deficiencias, "que deben abundar en México". ${ }^{42}$ Se esperaba que se estudiaran esos casos en un espacio clínico controlado, como un laboratorio, en un número de tres a seis casos. La FR empezó a interesarse más en el aspecto clínico de la nutrición, enfocado en el individuo, puesto que podían obtenerse resultados visibles y más rápidos gracias a intervenciones puntuales, similares a los programas que habían desarrollado con éxito en México para la erradicación de enfermedades como la anquilostomiasis. Un enfoque más integral, como lo que proponía la nutrición social, y que incluía el estudio detallado y más complejo de las poblaciones, empezó a ser cuestionado a inicios de la década de 1940, ante la inminencia del final de la guerra en Europa. Las palabras de Ferrell, escritas en 1943, concluían con la sugerencia de una nueva línea de

41 "Program for Nutrition Work in Mexico", 1943, RAC, RFR, RG 1.1, S 323, B 12, F 81.

${ }^{42}$ Ferrell a Payne, 13 de julio de 1943, RAC, RFR, RG 1.1, S 323, B 12, F 81. 
investigación para Payne, lo que resultaría premonitorio: “No estoy sugiriendo [que se lleve a cabo] en este momento este programa [de enfocarse en los casos clínicos], pero estoy indicando que las tendencias de las discusiones en este momento sugieren esa posibilidad". ${ }^{43}$

De Paula Miranda, a la cabeza del INN, fue un buen administrador de las investigaciones individuales que los organismos internacionales requerían. Fue el nodo por el cual la investigación sobre alimentación en México que requerían los organismos filantrópicos (como la FR o la FK) o internacionales (como la OsP, la FAO y la Organización Mundial de la Salud [OMs], fue asociada con el InN. Sin embargo, el trabajo de De Paula Miranda en el INN fue minado por la fragmentación de labores y los compromisos que había adquirido, además de que el estudio de las poblaciones, privilegiado en el periodo de entreguerras, cedió el paso al estudio de la producción agrícola como principal "arma” contra el "hambre" en los nuevos organismos internacionales de la segunda posguerra. ${ }^{44}$ Este enfoque, el aumento en la productividad, era el defendido por la NSD de la FR, orientando la investigación gradualmente hacia ese ámbito. La nutrición social predicada por Miranda y el INN perdieron terreno rápidamente en el ambiente de cambios de la posguerra.

Otros problemas surgieron a su vez por la falta de recursos a las diferentes áreas de investigación. El laboratorio de análisis de alimentos, en parte financiado por la FK, había surgido por la presencia de Robert S. Harris, del MIT. De esta colaboración se publicaron las tablas de composición de alimentos mexicanos del InN. Sin embargo, una visita de Harris a México, a inicios de 1947, mostró la dramática situación en que operaba el recién construido edificio del INN: no había tarjas en los laboratorios,

43 Ferrell a Payne, 13 de julio de 1943, RAC, RFR, RG 1.1, S 323, B 12, F 81. ${ }^{44}$ En la Conferencia de 1943, la nutrición fue considerado un arma contra la guerra y para preservar la paz. Véase VARGas-Domínguez, "Entre la nación y el mundo". 
ni campanas de extracción de gases, ni agua corriente; las conexiones para gas presentaban fugas y, para repararlas, como la instalación había quedado debajo de capas de cemento, había que romper paredes para su compostura. Esta complicada situación retrasaba los análisis requeridos. Además de estos problemas materiales, había otros de tipo económico. El pago de los investigadores del laboratorio había formado parte del convenio con la KF por medio de la osp, sin embargo, al finalizar su vigencia, De Paula Miranda se vio imposibilitado para pagar el tiempo completo a su personal, sino solo medio tiempo. Aunado a lo anterior, a partir de 1947, la carga financiera del INN se vio incrementada por el retiro de fondos internacionales. Los fondos debían ir a otra institución que fuese viable para realizar trabajos de tiempo completo; en su caso, recomendaba Harris, el Hospital de Enfermedades de la Nutrición, a cargo de Salvador Zubirán. ${ }^{45}$

Otro problema provino de quienes habían fortalecido mucho el trabajo del INN, es decir, la IHD de la FR. Desde el final de la segunda guerra mundial y el surgimiento de la oms, la directiva de la FR entendió que la OMs era la heredera del trabajo de la IHD. Los proyectos de la IHD fueron clausurados paulatinamente y la IHD cerró en 1951, lo que llevó al fin de una era en la salud pública. ${ }^{46}$ El INN, a cargo de De Paula Miranda, y dada su vinculación con el análisis de granos de la NSD y sus lazos con la IHD, había intentado equilibrar la cooperación con ambas divisiones y por un tiempo logró reconciliar en su instituto las dos perspectivas sobre la alimentación que en ese momento parecían incompatibles: el mantenimiento del estudio de poblaciones locales y sus

\footnotetext{
45 Harris a Soper, 20 de febrero de 1947, RFR, RG 1.1, S 323, B 12, Folder 83. 46 Matysiak, Health $\&$ Well-being, pp. 171-173. De acuerdo con Matysiak, el cierre de la IHD coincidió con el auge del programa de alimentación del PAM. Sin embargo, parece ser que el cierre de la IHD fue más bien una pugna entre el programa de la IHD y la NSD; este aspecto requiere ser profundizado en otras investigaciones.
} 
requerimientos específicos por la IHD, y que eran compartidos por los investigadores nacionales, tanto médicos como antropólogos, y el enfoque de la NSD en la mejora de la productividad agrícola como solución a los problemas globales.

Este último enfoque tuvo el respaldo de la FAO, con énfasis en la producción y distribución de alimentos, aspecto fundamental para acabar con el hambre según esta organización. El PAM de la NSD prometía ser un ejemplo claro de que el futuro de la investigación debería enfocarse al aumento de la producción agrícola. En esta encrucijada, el laboratorio de análisis de los alimentos del INN se comprometió a estudiar la composición de los granos del PAM. ${ }^{47}$ Para ello, la IHD y la NSD cooperaron en la compra de instrumental del laboratorio en 1944, en una de las últimas colaboraciones de las dos divisiones. ${ }^{48}$

Poco después de las negociaciones para mantener el laboratorio a flote, De Paula Miranda renunció a su puesto como director del InN y fue sustituido por José Calvo de la Torre. No es claro el porqué de la renuncia, pero podemos aventurar que fue por su precario estado de salud. Después de esto, la capacidad de negociación que De Paula Miranda había cultivado se vio comprometida. Él murió en 1951, y su proyecto de nutrición social en el INN se sostuvo solo por unos años más.

El doctor José Calvo de la Torre había sido becario de la FR en Administración de la Salud Pública en el Hospital Johns Hopkins de Estados Unidos, e inició su trabajo en el INN en 1943. Calvo recibía su sueldo directamente de la FR y junto con otros miembros del INN, estaba comprometido con el trabajo de De Paula Miranda y el desarrollo de las investigaciones requeridas por la FR. Gracias a las buenas relaciones de Calvo con Harrar, de la NSD, en 1948 logró reactivar los proyectos de nutrición,

47 Anderson, 11 de septiembre de 1947, RAC, RFR, RG 1.1, S 323, B 12, F 83. ${ }^{48}$ Smith a Downs, 22 de septiembre de 1947, RAC, RFR, RG 1.1, S 323, B $12, \mathrm{~F} 83$. 
pero enfatizando el análisis bromatológico, es decir, el aspecto bioquímico. Durante la gestión de Calvo, el vínculo con el PAM se fortaleció, consolidando la sección de bromatología, la que se hacía cargo del análisis de las semillas creadas en el PAM. A pesar de que tenía mayor énfasis el estudio de los granos para seleccionar los más productivos, parte del enfoque de nutrición social del InN se mantuvo. Calvo proyectó la creación de Brigadas Culturales de Alimentación "con el fin de estudiar sus problemas de alimentación y de nutrición [del pueblo] y [que] realicen al mismo tiempo la correspondiente labor educativa". Calvo pretendía también incrementar el número de médicos encuestadores y de trabajadoras sociales del Instituto para extender la cobertura de las encuestas de alimentación hacia el resto de la República y formar lo que llamaba la "geografía dietética del país". ${ }^{49}$ Estos proyectos al parecer no se llevaron a cabo.

Como ya he mencionado, la creación en 1949 del INCAP congregó a muchos de los actores regionales en el terreno de la nutrición, y la cooperación de las agencias surgidas de la posguerra orientaron sus fondos al funcionamiento de este instituto en Guatemala, y los retiraron del instituto mexicano, ${ }^{50}$ a pesar de que había sido tomado como el referente a seguir. Por ejemplo, en 1949, funcionarios de la OSP, la FR, la FK, así como Robert S. Harris, del MIт, junto con Calvo de la Torre, discutieron los programas generales tanto del INN como del INCAP, así como las actividades a desarrollar en los siguientes años. De Paula Miranda, para la fecha de estas discusiones, solo participó con una ponencia menor en dicha reunión..$^{51}$

Con el surgimiento del INCAP, el INN se enfrentó a un retiro de los fondos internacionales, lo cual acentuó los problemas que se habían agudizado con la muerte de De Paula Miranda. Entre los

49 Calvo, “Instituto Nacional de Nutriología de México”, pp. 1247-1252.

50 Pernet, "Between Entanglements and Dependencies".

51 “Nutrición”, p. 1182. 
problemas estaban la falta de coordinación y el desconocimiento de lo hecho dentro de Nutriología. Por ejemplo, el Comité de Enlace de México ante la FAO, que había estado a cargo de De Paula Miranda como representante de la Secretaría de Salud y de la Comisión Nacional de Alimentación, quedó sin cabeza por un tiempo. Previo a una reunión de la Asamblea de Ministros de Agricultura del Continente Americano de la FAO para México, Caribe y la América Central en 1953, se "descubrió" que el Comité de enlace no era competencia de la Secretaría de Agricultura, sino de la Secretaría de Salud. Quien realizó este "descubrimiento" fue Jesús Díaz Barriga, y proponía que se creara de nueva cuenta una Comisión Nacional de Alimentación, encabezada por el representante de la Secretaría de Salud, que él mismo ocuparía. No se creó una nueva comisión, pero Díaz Barriga, con José Quintín Olascoaga y Juana Navarro, investigadores también de Nutriología, formaron parte de la Junta Nacional para el Mejoramiento de la Alimentación, a cargo del INN. ${ }^{52} \mathrm{~A}$ pesar de que parecía que el INN se mantenía en el centro de los proyectos de alimentación locales, en realidad iba perdiendo relevancia.

El trabajo educativo del INN tampoco fue lo que se esperaba por parte de los circuitos internacionales que lo habían financiado. Por ejemplo, durante 1946, Edna Noble White, interesada en la educación infantil y en la economía doméstica,

52 Jesús Díaz Barriga, junto con su esposa y su hermana, trabajaban en el INN, y promovían el cultivo de soya y su adición a los alimentos, e hicieron recetarios para crear dietas "mejoradas" por la soya. Cabe hacer la aclaración de que eran parte de Nutriología, no de Nutrición, como aparece en Fernández Aceves. Lo anterior como ejemplo de la confusión entre Nutriología con su sucesor Nutrición. Fernández Aceves, "Política y ciudadanía", pp. 91-95. Los trabajos de la familia de Díaz Barriga son mencionados por José Calvo de la Torre, "Historia, programa y personal del Instituto Nacional de Nutriología”, AHSSA, SSA, SubSyA, C 21, E 2. Es necesario hacer la historia de esta Junta Nacional de Alimentación dado que aún no ha sido explorado su papel en el desarrollo de las políticas de alimentación en México. 
visitó varios centros de salud en México. En un reporte hecho para la FR, aclaraba el carácter de los cursos de nutrición otorgados por Nutriología, y que eran impartidos en el Instituto Nacional de Cardiología. Los cursos, de acuerdo con ella, eran de mala calidad, sin práctica hospitalaria, y ofrecían un panorama desolador, dados los bajos salarios y los pocos espacios de trabajo para quienes lograban graduarse. En su reporte, White mencionaba que las condiciones podrían mejorar en el futuro, con la construcción del edificio para el Instituto, aunque esto al parecer no se llevó a cabo. ${ }^{53}$ Después de los problemas antes mencionados, como la muerte de De Paula Miranda, el cierre de los recursos internacionales, las disputas internas en los organismos que los financiaban, y el cambio de intereses en la investigación, además de los problemas de coordinación y de investigación internos del Instituto, el INN cerró sus puertas en 1956, y con él, el énfasis en el aspecto social de la nutrición. Finalizaba, así, uno de los capítulos de la nutrición en México, y con él, esta etapa de colaboración internacional. Este instituto, así como este periodo, ofrece aún vetas por explorar, como, por ejemplo, las trayectorias de los investigadores que laboraron en el INN y el impacto de proyectos como los Comedores en la vida de las personas que fueron asistidas ahí. También es necesario plantearse la vinculación entre las empresas productoras de alimentos con los investigadores del Instituto, así como también las redes de intercambios académicos con otros países de Latinoamérica, no solo con Estados Unidos. Asimismo, falta aún un estudio que explore en mayor profundidad el vínculo entre las políticas alimentarias de México después de la posguerra y la forma como se argumentó la necesidad de intervenciones agrícolas orientadas a una mayor productividad. El vínculo entre salud y alimentación, aunque retomado una y otra vez, sigue siendo empleado para argumentar políticas públicas de salud.

53 Edna White, RAC, RFR, RG 2-1947, S 323, B 379 F 2559. 


\section{EPÍLOGO}

En 1956, Richmond K. Anderson, de la FR, realizó de nueva cuenta una visita a México y, ante la desaparición de Nutriología, se preguntaba cuál sería el destino del personal y la investigación ahí realizada. Calvo de la Torre conversó con Anderson sobre las posibles alternativas a seguir. Cabía la posibilidad de que lo que quedaba del INN se integrase a uno de los institutos descentralizados que existían en ese momento con interés en la nutrición: el Hospital Infantil, el Instituto de Cardiología o el Hospital de Enfermedades de la Nutrición. La opinión de Anderson sobre este último era la siguiente: "es una buena institución, pero en realidad es un hospital general y de cirugía, que otorga algo de atención a la nutrición más que un hospital de nutrición per $s e$ ". ${ }^{54}$ Posteriormente, Anderson se entrevistó con René Cravioto, otro investigador del INN, quien le mencionó que, si se quería resucitar al INN, se debía integrar al Hospital Infantil dirigido por Federico Gómez. De acuerdo con Cravioto, existía el riesgo de que Salvador Zubirán, del Hospital de Enfermedades de la Nutrición, "con orientación clínica”, quisiera absorber a $\mathrm{Nu}$ triología, lo cual "podría tener alguna ventaja, pero él [Cravioto] duda de que, en lo general, sea para bien" ${ }^{55}$ De acuerdo con las memorias de Anderson y su entrevista con Cravioto, De Paula Miranda nunca se había llevado bien con Zubirán e incluso habían peleado entre ellos. A pesar de estas reticencias, lo que quedaba del INN pasó a formar parte del Hospital de Enfermedades de la Nutrición, donde la perspectiva clínica, el "futuro" que había sido propuesto por Ferrell en 1943, prevaleció.

En 1956, año de cierre de Nutriología, el Hospital de Enfermedades de la Nutrición, a petición de Salvador Zubirán, cambió de nombre y empezó a conocerse como Instituto Nacional

\footnotetext{
${ }^{4}$ Anderson, marzo 1956, RAC, RFR, Officer's diaries, RG 12, Box 6.

55 Anderson, marzo 1956, RAC, RFR, Officer's diaries, RG 12, Box 6.
} 
de Nutrición. Para la justificación del cambio de nombre se dijo que la Secretaría de Salubridad y Asistencia debería cambiar el uso de "hospital" por el de "instituto", además de "generalizar el concepto de enfermedades de la nutrición por el concepto exclusivo de nutrición". ${ }^{56}$ El cambio de denominación fue crucial. El Hospital dejaba su papel más restringido al estudio de las enfermedades y adquirió así parte de los compromisos de salud pública y nutrición social que había mantenido el INN, manteniendo entre sus funciones el estudio del vínculo existente entre el aspecto socioeconómico y la alimentación, la educación de la población en temas de nutrición, la investigación de los alimentos locales en su composición y la colaboración con los centros de producción agrícola, así como la cooperación con instituciones internacionales especializadas en la alimentación. ${ }^{57}$

Un epílogo un tanto sombrío del trabajo del INN fue el desconocimiento de los esfuerzos de investigación que se llevaron a cabo en él durante sus más de diez años de funcionamiento. De acuerdo con Cárdenas de la Peña, en 1957 el nuevo Instituto de Nutrición propuso a Ignacio Morones Prieto, entonces a la cabeza de la Secretaría de Salubridad y Asistencia, la elaboración de un "ensayo piloto" para evaluar a grupos poblacionales en el aspecto nutriológico. La primera fase propuesta para este ensayo fue el "adiestramiento de personal", cuya necesidad se argumentó de la siguiente manera: "en nuestro país no existen nutriólogos con conocimientos de las ciencias básicas de la nutrición, los aspectos sanitarios de esta ciencia y los relacionados con la valoración estadística de los datos obtenidos", ${ }^{58}$ una afirmación cuestionable, dado el amplio trabajo de encuestas de nutrición que se había desarrollado en el INN hasta su cierre, olvidando también los esfuerzos internacionales que habían

56 Ricardo Garza Galindo citado por Cárdenas de la PeÑa, Enlace SZ-INN: crónica de un instituto, p. 200.

57 CÁrdenas de la PeÑa, Enlace SZ-INN: crónica de un instituto, p. 201.

58 Cárdenas de la PeÑa, Enlace SZ-INN: crónica de un instituto, p. 202. 
usado esta herramienta..$^{59}$ Las encuestas de nutrición, que también evaluaban el aspecto socioeconómico, no fueron tan valoradas en la nueva faceta del Hospital de Enfermedades que entonces empezaba a ser conocido como Instituto. Este nuevo Instituto, a cargo de Zubirán, se fundó asumiendo que no había nutriólogos en México, por lo que se requería que se entrenara personal en otros países, en "Guatemala y en Lima, dentro de los centros especializados donde observen los sistemas de trabajo de campo, encuestas sanitarias, estudios clínicos en grupos de población, métodos especializados de laboratorio y demás". 60 El conocimiento especializado local fue olvidado o descartado en favor del conocimiento externo. El centro que se convirtió en el más importante en el ámbito regional para el estudio de la nutrición fue el INCAP en Guatemala, y recibió a los nuevos investigadores mexicanos, a pesar de que hasta hacía unos años, el entrenamiento de los nutriólogos del INCAP se había realizado en México, en el InN. Una nueva etapa se abría en el estudio de la nutrición, en donde la nutrición social se subordinaba a la investigación clínica de las enfermedades de la nutrición y al estudio bioquímico de los alimentos. El problema de la nutrición dejó de ser, por un tiempo, un asunto social, sino clínico, y la posible solución, fuera del ámbito médico, fue aumentar la productividad agrícola. Este enfoque productivo perseguido por el PAM, sí se consideró un éxito: las semillas analizadas en el INN se convirtieron posteriormente en los detonadores de lo que sería llamada la revolución verde, que se entendió como un programa de éxito agrícola. Podríamos decir que la semilla de este movimiento surgió de México y se analizó en el INN, a costa de la comprensión integral de la nutrición que predicaban los proyectos de nutrición social.

59 YÁÑEZ ANDrade, "El problema de la alimentación”, pp. 66-97.

60 CÁrdenas de la PeÑa, Enlace SZ-INN: crónica de un instituto, p. 202. 


\section{SIGLAS Y REFERENCIAS}

AGN, SEP,DPH Archivo General de la Nación, fondo Secretaría de Educación Pública, Departamento de Psicopedagogía e Higiene, Ciudad de México, México

AGN, $P, M A C$ Archivo General de la Nación, fondo Presidentes, Manuel Ávila Camacho, Ciudad de México, México

AHANMM, CE Archivo Histórico de la Academia Nacional de Medicina de México, Colección Expedientes, Ciudad de México, México

AHSSA, SSA, SubSyA Archivo Histórico de la Secretaría de Salud, fondo Secretaria de Salubridad y Asistencia, Subsecretaria de Salubridad y Asistencia, Ciudad de México, México

AHSSA, BP, D, SS Archivo Histórico de la Secretaría de Salud, fondo Beneficencia Pública, Dirección, Subsecretaria, Ciudad de México, México

CNA Comisión Nacional de Alimentación

FAO Organización para la Agricultura y la Alimentación de las Naciones Unidas

FK Fundación W. K. Kellogg

FR Fundación Rockefeller

INCAP Instituto de Nutrición de Centro América y Panamá

IHD International Health Division

INN Instituto Nacional de Nutriología

NSD Natural Sciences Division

OEE Oficina de Estudios Especiales

OES Oficina de Especialización Sanitaria

OMS Organización Mundial de la Salud

OSP Oficina Sanitaria Panamericana

PAM Programa Agrícola Mexicano

RAC, RFR Rockefeller Archive Center, Rockefeller Foundation Records, Tarrytown, Nueva York, Estados Unidos

SAP Secretaría de Asistencia Pública

Agostoni, Claudia, "Médicos rurales y medicina social en el México posrevolucionario (1920-1940)", en Historia Mexicana, LXIII: 2 (250) (oct.-dic. 2013), pp. 745-801.

Aguilar Rodríguez, Sandra, "Cooking Modernity: Nutrition Policies, Class, and Gender in 1940s and 1950s Mexico”, en The Americas, 64: 2 (2007), pp. 177-205. 
Aguirre Beltrán, Gonzalo, Antropología médica, México, Centro de Investigaciones y Estudios Superiores en Antropología Social, Ediciones de la Casa Chata, 1986.

Anderson, Richmond K., José Calvo de la Torre, et al. "Nutrition appraisals in Mexico", en American Journal of Public Health, $38: 8$ (ago. 1948), pp. 1126-1135.

Barona, Josep Lluís, "Nutrition and Health. The International Context During the Interwar Crisis", en Social History of Medicine, 21: 1 (abr. 2008), pp. 87-105.

Barona, Josep Lluís, The Problem of Nutrition. Experimental Science, Public Health, and Economy in Europe, 1914-1945, Bruselas, Berna, Fránkfurt, Nueva York, Peter Lang, 2010.

BIRN, Anne-Emanuelle, Marriage of Convenience: Rockefeller International Health and Revolutionary Mexico, Rochester, Nueva York, University of Rochester Press, Serie Rochester Studies in Medical History, 2006.

BuschinI, José, "La alimentación como problema científico y objeto de políticas públicas en la Argentina: Pedro Escudero y el Instituto Nacional de la Nutrición, 1928-1946”, en Apuntes, 79 (2016), pp. 129-156.

Calvo de la Torre, José, "Instituto Nacional de Nutriología de México", en Boletín de la Oficina Sanitaria Panamericana, 28: 12 (dic. 1949), pp. 1247-1252

Cárdenas de la PeÑa, Enrique, Enlace SZ-INN: crónica de un instituto, México, Instituto Nacional de la Nutrición “Salvador Zubirán”, 1991.

Carrillo, Ana María, "Salud pública y poder en México durante el Cardenismo, 1934-1940”, en DYNAMIS. Acta Hisp. Med. Sci. Hist. Ilus., 25 (2005), pp. $145-178$.

Совв, William C., "The historical backgrounds of the Mexican Agricultural Program (annotated edition)", en Rockefeller Foundation Records, 90, Rockefeller Archive Center, 1956.

Cravioto, René O., Ernest E. Lockhart, Richmond K. Anderson, Francisco de Paula Miranda, Robert S. Harris, Estela Aguilar, Elizabeth W. Tapia, et al. "Composition of Typical Mexican Foods", en The Journal of Nutrition, 29: 5 (mayo 1945), pp. 317-329. 
Cravioto, René O., Ernest E. Lockhart, Richmond K. Anderson, Francisco de Paula Miranda y Robert S. Harris, "Nutritive Value of the Mexican Tortilla”, en Science, 102: 2639 (jul. 1945), pp. 91-93.

Cravioto, René O., Guillermo Massieu H., Jesús Guzmán G. y José Calvo DE la Torre, "Composición de alimentos mexicanos", en Ciencia, xI: 5-6 (1951), pp. 129-155.

Cueto, Marcos y Steven Palmer, Medicine and Public Health in Latin America, Nueva York, Cambridge University Press, 2014.

Cueto, Marcos, Missionaries of Science: The Rockefeller Foundation and Latin America, Bloomington, Indiana University Press, Philanthropic Studies, 1994.

Cullather, Nick, The Hungry World: America's Cold War Battle against Poverty in Asia, Harvard University Press, 2010.

Departamento de Salubridad Pública, Memoria Anual del Departamento de Salubridad Pública correspondiente al periodo 1 septiembre 1942-31 agosto 1943, México, Departamento de Salubridad, 1943.

Fernández Aceves, María Teresa, "Política y ciudadanía: el liderazgo de María Guadalupe Urzúa en la Confederación Nacional Campesina, 1953-1957”, en Relaciones. Estudios de Historia y Sociedad, 38 (2017), pp. 71-100.

Lorenzo Río, María Dolores, El Estado como benefactor. Los pobres y la asistencia pública en la ciudad de México, México, El Colegio de México, El Colegio Mexiquense, 2011.

Magaña Valladares, Laura y María Rosa Gudiño-Cejudo, Reseña histórica de la Escuela de Salud Pública de México. Noventa años formando salubristas e investigadores para mejorar la salud de la población, Cuernavaca, Morelos, Instituto Nacional de Salud Pública, 2012.

Matysiak, Angela, Health \& Well-being: Science, Medical Education, and Public Health, Nueva York, The Rockefeller Foundation, Rockefeller Foundation Centennial Series, 2014.

“Nutrición”, Sección Notas y Revistas, en Boletín de la Oficina Sanitaria Panamericana, año 28, 11 (nov. 1949), p. 1182. 
Pernet, Corinne A., "Between Entanglements and Dependencies: Food, Nutrition, and National Development at the Central American Institute of Nutrition (INCAP)", en Marc Frey, Sönke Kunkel y Corinna R. Unger, (eds.), International Organizations and Development, 1945-1990, pp. 101-125, Palgrave Macmillan, Transnational History Series, 2014.

“El Primer Congreso Indigenista Interamericano”, en Boletín Bibliográfico de Antropología Americana (1937-1948), 4: 1 (1940), pp. 1-36.

Quintín Olascoaga, José, Nutriología, México, 1945.

Rodríguez de Romo, Ana Cecilia y Martha Eugenia Rodríguez Pérez, "Historia de la salud publica en México: siglos xix y xx", en História, Ciências, Saúde-Manguinhos, 5: 2 (1998), pp. 293-310.

Scrimshaw, Nevin S., "Estudios sobre los problemas de la nutrición en la América Latina”, en Boletín de la Oficina Sanitaria Panamericana, año 28, 12 (dic. 1949), pp. 1201-1214.

Smith, David F., "Nutrition Science and the Two World Wars", en Nutrition in Britain. Science, scientists and politics in the twentieth century (Studies in the Social History of Medicine), Londres y Nueva York, Routledge, 1997, pp. 142-165.

VARgas-Domínguez, Joel, “Entre la nación y el mundo: la nutrición en México en la Conferencia de las Naciones Unidas sobre alimentación de 1943", en Cuestión social, politicas sociales y construcción del Estado Social en América Latina en los siglos XIX y XX, editado por Mario BARBOSA CRUZ y Fernando Remedi, México, Córdoba, Argentina, Universidad Autónoma Metropolitana-Cuajimalpa, Centro de Estudios Históricos Carlos Segreti, 2014, pp. 175-192.

VARgas-Domínguez, Joel, "El metabolismo racial: estudios eugenésicos en Jamaica y Yucatán entre 1920 y 1940”, en Revista Ciencias de la Salud, 13, (2015), pp. 85-103.

Vargas-Domínguez, Joel, "La construcción del Instituto Nacional de Nutriología en México: Conexiones locales y globales”, en Edna María SuÁrez Díaz y Gisela Mateos (eds.), Aproximaciones a lo local y lo global: América Latina en la historia de la ciencia contemporánea, México, Centro de Estudios Filosóficos, Políticos y Sociales Vicente Lombardo Toledano, 2016, pp. 155182. 
Vargas-Domínguez, Joel, “The 'problematic' Otomi: Metabolism, nutrition, and the classification of indigenous populations in Mexico in the 1930's.", en Perspectives on Science, 25: 5 (sep.-oct. 2017).

Vargas-Domínguez, Joel y Adriana Minor García, "Mexican Scientists in the Making of Nutritional and Nuclear Diplomacy in the First Half of the Twentieth Century", en HoST-Journal of History of Science and Technology, 11: 1 (2017), pp. 34-56.

Vernon, James, Hunger. A Modern History, Cambridge, Mass., Londres, The Belknap Press of Harvard University Press, 2007.

Viesca Treviño, Carlos, "La Gota de Leche. De la mirada médica a la atención médico-social en el México posrevolucionario", editado por Claudia Agostoni, México, Universidad Nacional Autónoma de México, Benemérita Universidad Autonoma de Puebla, 2008, pp. 195-256.

YÁÑEz Andrade, Juan Carlos, "El problema de la alimentación: un enfoque desde las encuestas de nutrición (Chile, 1928-1938)", en América Latina en la Historia Económica, 24 (2017), pp. 66-97.

Zubirán, Salvador, Mi vida y mi lucha. Autobiografía, México, Fundación Mexicana para la Salud, Editorial Médica Panamericana, 1996. 
